\title{
Electron Spins in Artificial Atoms and Molecules for Quantum Computing *
}

\author{
Vitaly N. Golovach and Daniel Loss \\ Department of Physics and Astronomy, University of Basel, \\ Klingelbergstrasse 82, CH-4056 Basel, Switzerland
}

(October 28, 2018)

\begin{abstract}
Achieving control over the electron spin in quantum dots (artificial atoms) or real atoms promises access to new technologies in conventional and in quantum information processing. Here we review our proposal for quantum computing with spins of electrons confined to quantum dots. We discuss the basic requirements for implementing spin-qubits, and describe a complete set of quantum gates for single- and two-qubit operations. We show how a quantum dot attached to leads can be used for spin filtering and spin read-out, and as a spin-memory device. Finally, we focus on the experimental characterization of the quantum dot systems, and discuss transport properties of a double-dot and show how Kondo correlations can be used to measure the Heisenberg exchange interaction between the spins of two dots.
\end{abstract}

\section{INTRODUCTION}

Coulomb blockade phenomena have atracted much interest during the last few dacades [1]. Creation of confined electron systems at the nanometer scale has made it possible to study the quantum-meachnical nature of the band electron in a variety of materials. The tunability of the quantum-dot devices provided a unique opportunity to study the charging effects, and hence, the correlation effects associated with the Coulomb charging energy. However, in confined systems of smaller sizes, where the size-quantization energy is resolved, new correlations set in due to the Pauli exclusion principle. This brings along the electron spin as a degree of freedom in quantum confined structures, and accessing it in a deterministic way would allow for novel implementations in quantum information processing. An increasing number of spin-related experiments [2] indeed show that the spin of the electron in quantum-confined nanostructures is a promising candidate for information processing, due to the unusually long (100's nanosecs) spin dephasing times [3 5. On the other hand there are propsed methods [8] for efficient and deterministic control of the spin state in single quantum dots as well as methods of entangling the spins of two dots, the latter being a crucial element in quantum information processing. Thus, the field of interest falls into two parts, one being improving the technologies for conventional computation, and the other - implementing fundamentally new algorithms of computation with quantum bits of information (qubits) and devising a scalable quantum computer in the long run. In conventional computers, the electron spin can be expected to enhance the performance of quantum electronic devices, such as spin-transistors (based on spin-currents and spin injection), non-volatile memories, single spin as the ultimate limit of information storage etc. [2,9]. For implementing quantum computing 10, as first pointed out in Ref. [8], the spin of a confined electron appears as the most natural candidate for the qubit. Indeed, provided the spinorbit coupling is negligible, the intrinsic two-state space of the spin encodes exactly one qubit and allows for no undesired parts of the Hilbert space, transitions to which could lead to leakage errors in quantum computation. We have shown 80 that the spin qubits, when located in quantum-confined structures such as semiconductor quantum dots or atoms or molecules, satisfy all requirements needed for a scalable quantum computer.

The long distances, of up to $100 \mu \mathrm{m}$ [3], over which spins can be transported phase-coherently, make the electron spin a plausible candidate for quantum information transmission in solid state devices. A spin-qubit attached to a mobile electron can be transported along conducting wires between different subunits in a quantum network 11, 12]. Entangled electrons, which can be created in coupled quantum dots or via a superconductor 13, provide a source of Einstein-Podolsky-Rosen (EPR) pairs 11, 12, wich are necessary for secure quantum communication.

The methods used to implement the electron spin in conventional computers and in quantum computers are often identical, because of the quantum-mechanical nature of the electron and its spin. Our short-term goal is to find ways to control the coherent dynamics of electron spins in quantum-confined nanostructures. The use of solid state physics as a base for implementing the quantum computer is motivated by the unparalleled flexibility in designing an appropriate medium for the realization of a given physical phenomena.

In the following, we review the status of our theoretical efforts towards the goal of implementing quantum computation with electron spins in quantum-confined nanostructures.

\footnotetext{
*Invited review prepared for Special Issue of Semiconductor Science and Technology, "Semiconductor Spintronics", ed. H. Ohno, 2002.
} 


\section{A. Quantum Computing and Quantum Dots}

The possibility of outperforming classical computation, which opens up in quantum algorithms such as the one discovered by Shor [14] and by Grover [15], has attracted much interest. A quantum algorithm makes use of the quantum computers's ability to exist in any superposition of the states of its binary basis and to perform quantum time evolution for computation; hence the parallelism of quantum computing. The requirement for the quantum bit of information (qubit), which is at the heart of the quantum computer, is that it can exist in any state of a quantum two two-level system, i.e. $|\psi\rangle=\alpha|0\rangle+\beta|1\rangle$, where $|0\rangle$ and $|1\rangle$ are the states of the "classical" bit, and $|\alpha|^{2}+|\beta|^{2}=1$. Apart from this, a qubit should be able to couple to any other qubit in the quantum comupter and form a coherent two-qubit state. These two elements, which are the one- and two-qubit gates, are sufficient for forming a many-qubit coherent state and implementing any quantum algorithm.

A recently growing list of quantum tasks [11, 16] such as cryptography, error correcting schemes, quantum teleportation, etc. have indicated even more the desirability of experimental implementations of quantum computing. On the other hand, there is also a growing number of proposed physical implementations of qubits and quantum gates. A few examples are: Trapped ions 117, cavity QED [18], nuclear spins 19,20], superconducting devices [21 24], and our qubit proposal [8] based on the spin of the electron in quantum-confined nanostructures, and in particular in quantum dots with an all-electrical control of spin. Subsequent proposals such as 20,25,26 are based on the same principles as introduced in $[8]$ and reviewed herein.

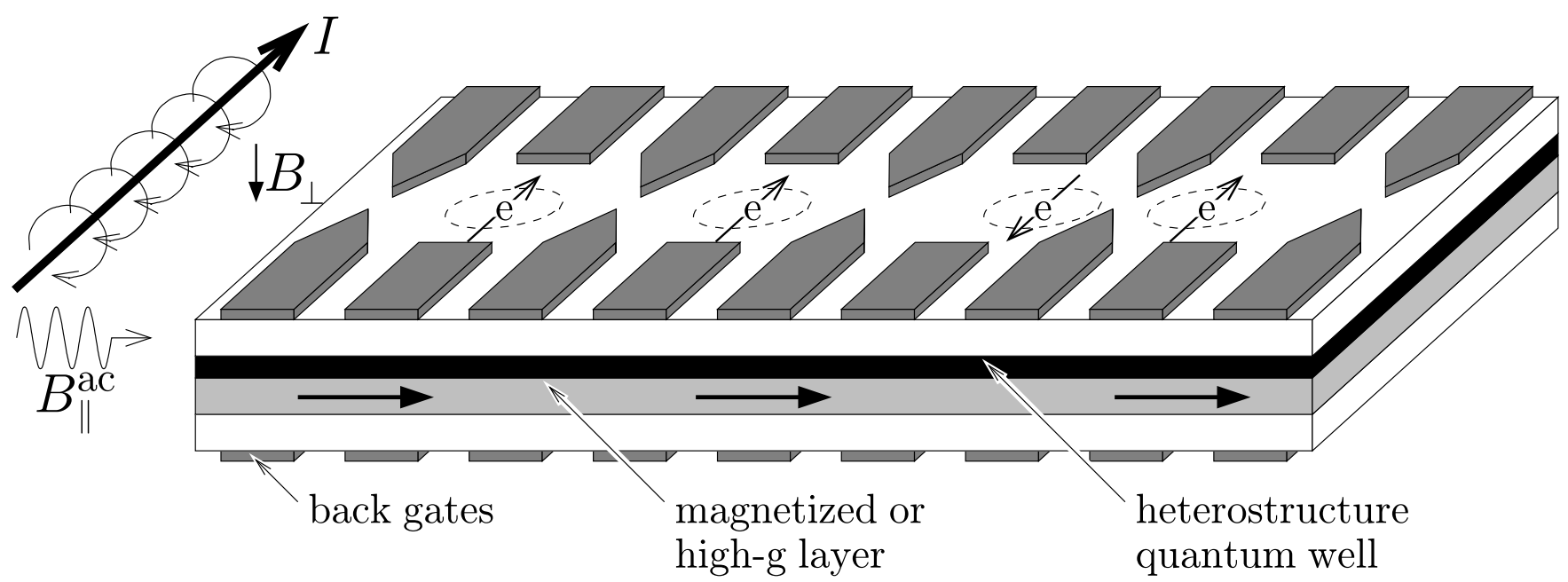

FIG. 1. Quantum dot array, controlled by electrical gating. The electrodes (dark gray) define quantum dots (circles) by confining electrons. The spin $1 / 2$ ground state (arrow) of the dot represents the qubit. These electrons can be moved by electrical gating into the magnetized or high- $g$ layer, producing locally different Zeeman splittings. Alternatively, magnetic field gradients can be applied, as e.g. produced by a current wire (indicated on the left of the dot-array). Then, since every dot-spin is subjected to a different Zeeman splitting, the spins can be addressed individually, e.g. through ESR pulses of an additional in-plane magnetic ac field with the corresponding Larmor frequency $\omega_{\mathrm{L}}=g \mu_{B} B_{\perp} / \hbar$. Such mechanisms can be used for single-spin rotations and the initialization step. The exchange coupling between the quantum dots can be controlled by lowering the tunnel barrier between the dots. In this figure, the two rightmost dots are drawn schematically as tunnel-coupled. Such an exchange mechanism can be used for the XOR gate operation involving two nearest neighbor qubits. The XOR operation between distant qubits is achieved by swapping (via exchange) the qubits first to a nearest neighbor position. The read-out of the spin state can be achieved via spin-dependent tunneling and SET devices [8], or via a transport current passing the dot 27]. Note that all spin operations, single and two spin operations, and spin read-out, are controlled electrically via the charge of the electron and not via the magnetic moment of the spin. Thus, no control of local magnetic fields is required, and the spin is only used for storing the information. This spin-to-charge conversion is based on the Pauli principle and Coulomb interaction and allows for very fast switching times (typically picoseconds). A further advantage of this all-electrical scheme is its scalability into an array of arbitrary size. 
Semiconductor quantum dots are structures where charge carriers are confined in all three spatial dimensions, the dot size being of the order of the Fermi wavelength in the host material, typically between $10 \mathrm{~nm}$ and $1 \mu \mathrm{m}$ [1]. The confinement is usually achieved by electrical gating of a two-dimensional electron gas (2DEG), possibly combined with etching techniques, see Fig. 11. Precise control of the number of electrons in the conduction band of a quantum dot (starting from zero) has been achieved in GaAs heterostructures 28. The electronic spectrum of typical quantum dots can vary strongly when an external magnetic field is applied [1,28], since the magnetic length corresponding to typical laboratory fields $B \approx 1 \mathrm{~T}$ is comparable to typical dot sizes. In coupled quantum dots Coulomb blockade effects [29], tunneling between neighboring dots [1, 29, and magnetization [30] have been observed as well as the formation of a delocalized single-particle state [31].

\section{GENERAL CONSIDERATIONS FOR QUANTUM COMPUTING WITH SPINS}

\section{A. Coherence}

Magneto-optical experiments, based on time-resolved Faraday rotation measurements, show long spin coherence times in doped GaAs in the bulk and a 2DEG [3]. At $B=0$ and $T=5 \mathrm{~K}$, a transverse spin lifetime (dephasing time) $T_{2}^{*}$ exceeding $100 \mathrm{~ns}$ was measured, with experimental indications that this time is a single-spin effect [3]. Since this number still includes inhomogeneous effects - e.g. g-factor variations in the material, leading to spins rotating with slightly different frequencies and thus reducing the total magnetization - it represents only a lower bound of the decoherence time $T_{2}$ of a single spin, $T_{2} \geq T_{2}^{*}$, which is relevant for using spins as qubits. Using the same pump-probe technique, spin dephasing times in semiconductor (CdSe) quantum dots have been measured [32], with at most one spin per dot. The relatively small $T_{2}^{*}$ dephasing times (a few ns at vanishing magnetic field), which have been seen in these experiments, probably originate from a large inhomogeneous broadening due to a strong variation of g-factors [32. Nevertheless, the fact that many coherent oscillations were observed 32 provides strong experimental support to the idea of using electron spin as a qubit.

\section{B. Upscaling}

To outperform a classical computer, a quantum computer will need a number of qubits on the order of $10^{5}$. Hence, it is essential that the underlying concept can be scaled up to a large number of qubits. This scaling requirement is, in principle, achievable with spinbased qubits confined in quantum dots, since producing arrays of quantum dots 11, 33 is feasible with today's technology of defining nanostructures in semiconductors. Of course, the actual implementation of such arrays (see Fig. 1) including all the needed circuits poses tremendous experimental challenges, but at least we are not aware of any physical restriction which would exclude such an upscaling for spin-qubits.

\section{Switching}

Quantum gate operations can be controlled through an effective Hamiltonian (see Sec. III and IV)

$$
H(t)=\sum_{i<j} J_{i j}(t) \mathbf{S}_{i} \cdot \mathbf{S}_{j}+\sum_{i} \mu_{B} g_{i}(t) \mathbf{B}_{i}(t) \cdot \mathbf{S}_{i} .
$$

The coupling constants $J_{i j}, g_{i}$ and the magnetic field (local) $\mathbf{B}_{i}$ are controlled via external gate fields, which are switched with some pulses $v(t)$. In the following we assume $J_{i j}$ to be non-zero only for the neighboring qubits. Note, however, that in cavity-QED systems there is also a long-range coupling of qubits [34], and that long-range coupling via a superconductor is also possible [35]. But even if the exchange coupling is only local, operations on non-neighboring qubits can still be performed. This is achieved by swapping states of neighboring qubits (see Sec. IV], which allows one to move the qubit around in an array of quantum dots and couple it to the desired other qubit.

For the gating mechanisms described in Sec. III and IV, only the time integral $\int_{0}^{\tau} P(v(t)) d t(\bmod 2 \pi)$ is important. Here, $P(v(t))$ stands for the exchange coupling $J$ or the Zeeman interaction. The requirement on the pulse shape is that it does not violate the validity of the effective Hamiltonian (11), but otherwise the gating mechanisms are independent of the actual shape of $v(t)$. Since the effective Hamiltonian (1) was obtained by projecting out higher energy states of one and two coupled dots (see Sec. IV A), care should be taken that the pulses do not excite the quantum dots to the projected-out energy levels. This can be achieved by switching $v(t)$ adiabatically, i.e. such that $|\dot{v} / v| \ll \delta \varepsilon / \hbar$, where $\delta \varepsilon$ is the energy scale on which excitations may occur. We find that $v(t)=v_{0} \operatorname{sech}(t / \Delta t)$, where $v_{0}$ is the pulse amplitude and $\Delta t$ the characteristic width, is optimal for a fast adiabatic switching, provided $1 / \Delta t \ll \delta \varepsilon / \hbar$. For a detailed analysis of adiabatic switching, see [36].

A single qubit operations can be performed for example in g-factor-modulated materials, as described in Sec. III. A spin can be rotated by a relative angle of $\varphi=\Delta g_{\text {eff }} \mu_{B} B \tau / 2 \hbar$ through changing the effective gfactor by $\Delta g_{\text {eff }}$ for a time $\tau$. Thus, a typical switching time for an angle $\varphi=\pi / 2$, a field $B=1 \mathrm{~T}$, and $\Delta g_{\text {eff }} \approx 1$ is $\tau_{s} \approx 30 \mathrm{ps}$. If slower operations are required, they are easily implemented by choosing a smaller $\Delta g_{\text {eff }}$, reducing the magnitude of the field $B$, or by replacing $\varphi$ by $\varphi+2 \pi n$ with integer $n$. 
Next we consider two exchange-coupled spins, which perform a square-root-of-swap gate for the integrated pulse $\int_{0}^{\tau_{s}} J(t) d t / \hbar=\pi / 2$, as described in Sec. IV. We apply a pulse $J(t)=J_{0} \operatorname{sech}\left(\left(t-\tau_{s} / 2\right) / \Delta t\right)$ with $J_{0}=$ $80 \mu \mathrm{eV}$, and choose $\Delta t=4 \mathrm{ps,} \mathrm{which} \mathrm{gives} \mathrm{for} \mathrm{the}$ switching time $\tau_{s} \approx 30 \mathrm{ps}$, and the adiabaticity criterion $\hbar / \Delta t \approx 150 \mu \mathrm{eV} \ll \delta \varepsilon$.

\section{Error Correction}

Realization of a reliable error-correction scheme 37. is one of the main goals in quantum computation. The known schemes for fault-tolerant quantum computation work if the gate operation error rate does not exceed a certain threshold value, usually about $10^{-4}$ (depending on the scheme) 38. If we take the ratio of the switching times from Sec. II G, $\tau_{s} \approx 30 \mathrm{ps}$, and the dephasing time from Sec. II A, $T_{2} \geq 100 \mathrm{~ns}$, we obtain a value close to this threshold. Thus, in our proposal, we can expect an arbitrary upscaling of the quantum computer, and we are no further limited by decoherence and lacking gate precision. We note that implementing an error-correction scheme requires a larger number of gate operations, and therefore, it is desirable to perform them in parallel; otherwise the pursued gain in computational power is used up for error correction. Hence, one favors concepts where a localized control of the gates can be realized such that operations can be performed in parallel. However, since there are still many milestones to reach before sophisticated error-correction schemes can be applied, one should by no means disregard setups where gate operations are performed in a serial way.

\section{SINGLE-SPIN ROTATIONS}

For quantum computing it is necessary (but not sufficient) to perform one-qubit operations. In the context of spin-qubits, it translates into single-spin rotations. This can be achieved by exposing a specific qubit to a timevarying Zeeman coupling $\left(g \mu_{B} \mathbf{S} \cdot \mathbf{B}\right)(t)$ 39, which can be controlled through both the magnetic field $\mathbf{B}$ and/or the g-factor $g$. Since only phases have a relevance, it is sufficient to rotate all spins of the system at once (e.g. by an external field $B$ ), but with a different Larmor frequency.

Localized magnetic fields can be generated with the magnetic tip of a scanning force microscope, a magnetic disk writing head, by placing the dots above a grid of current-carrying wires, or by placing a small wire coil above the dot etc.

Single-spin rotations can be achieved by ESR techniques 39]. One applies a static local magnetic field $B$ for the qubit(s), which should be rotated. An ac magnetic field is then applied perpendicular to the first field with the resonant frequency that matches the Larmor frequency $\omega_{L}=g \mu_{B} B / \hbar$. Due to paramagnetic resonance [40], this causes spin-flips in the quantum dots with the corresponding Zeeman splitting.

The equilibrium position of the electron can be moved around through electrical gating. Thus, if the electron wave function is pushed into a region with a different magnetic field strength or (effective) g-factor, one produces a relative rotation around the direction of $\mathbf{B}$ by an angle of $\varphi=\left(g^{\prime} B^{\prime}-g B\right) \mu_{B} \tau / 2 \hbar$, see Fig. 11. Regions with an increased magnetic field can be provided by a magnetic (dot) material while an effective magnetic field can be produced e.g. with dynamically polarized nuclear spins (Overhauser effect) [39].

We shall now explain a concept for using g-factormodulated materials 11, 33. In bulk semiconductors the free-electron value of the Landé g-factor $g_{0}=2.0023$ is modified by spin-orbit coupling. Similarly, the g-factor can be drastically enhanced by doping the semiconductor with magnetic impurities [5, 4]. In confined structures such as quantum wells, wires, and dots, the g-factor is further modified and becomes sensitive to an external bias voltage 41. We have numerically analyzed a system with a layered structure (AlGaAs-GaAs-InAlGaAsAlGaAs), in which the effective g-factor of electrons is varied by shifting their equilibrium position from one layer to another by electrical gating. We have found that in this structure the effective g-factor can be changed by about $\Delta g_{\text {eff }} \approx 1$ [33. Such a gate-controlled g-factor modulation has now been confirmed experimentally [42].

\section{TWO-QUBIT GATES}

The main component for every computer concept is a multi-(qu)bit gate, which eventually allows calculations through combination of several (qu)bits. Since two-qubit gates are (in combination with single-qubit operations) sufficient for quantum computation 43]-they form a universal set - we now focus on a mechanism that couples pairs of spin-qubits. Such a mechanism exists in coupled quantum dots, resulting from the combined action of the Coulomb interaction and the Pauli exclusion principle. Two coupled electrons in absence of a magnetic field have a spin-singlet ground state, while the first excited state in the presence of strong Coulomb repulsion is a spin triplet. Higher excited states are separated from these two lowest states by an energy gap, given either by the Coulomb repulsion or the single-particle confinement. The low-energy dynamics of such a system can be described by the effective Heisenberg spin Hamiltonian

$$
H_{\mathrm{S}}(t)=J(t) \mathbf{S}_{1} \cdot \mathbf{S}_{2}
$$

where $J(t)$ denotes the exchange coupling between the two spins $\mathbf{S}_{1}$ and $\mathbf{S}_{2}$, i.e. the energy difference between the triplet and the singlet. After a pulse of $J(t)$ with $\int_{0}^{\tau_{s}} d t J(t) / \hbar=J_{0} \tau_{s} / \hbar=\pi(\bmod 2 \pi)$, the time evolution $U(t)=T \exp \left(i \int_{0}^{t} H_{\mathrm{s}}(\tau) d \tau / \hbar\right)$ corresponds to the 
"swap" operator $U_{\text {sw }}$, whose application leads to an interchange of the states in qubit 1 and 2 [8]. While $U_{\mathrm{sw}}$ is not sufficient for quantum computation, any of its square $\operatorname{roots} U_{\mathrm{sw}}^{1 / 2}$, say $U_{\mathrm{sw}}^{1 / 2}|\phi \chi\rangle=(|\phi \chi\rangle+i|\chi \phi\rangle) /(1+i)$, turns out to be a universal quantum gate. Thus, it can be used, together with single-qubit rotations, to assemble any quantum algorithm. This is shown by constructing the known universal gate XOR [44], through combination of $U_{\mathrm{sw}}^{1 / 2}$ and single-qubit operations $\exp \left(i \pi S_{i}^{z} / 2\right)$, applied in the sequence [8],

$$
U_{\mathrm{XOR}}=e^{i(\pi / 2) S_{1}^{z}} e^{-i(\pi / 2) S_{2}^{z}} U_{\mathrm{sw}}^{1 / 2} e^{i \pi S_{1}^{z}} U_{\mathrm{sw}}^{1 / 2} .
$$

With these universal gates at hand, we can reduce the study of general quantum computation to the study of single-spin rotations (see Sec. III) and the exchange mechanism, in particular how $J(t)$ can be controlled experimentally. The central idea is that $J(t)$ can be switched by raising or lowering the tunneling barrier between the dots. In the following, we shall review our detailed calculations to describe such a mechanism. We note that the same principles can also be applied to other spin systems in quantum-confined structures, such as coupled atoms in a crystal, supramolecular structures, and overlapping shallow donors in semiconductors 20, 26 etc., using similar methods as explained below.

\section{A. Coupled Quantum Dots}

We consider a system of two tunnel-coupled quantum dots, achieved by gating a two-dimensional electron gas (2DEG), as described in Sec. IA. The dots are arranged in a plane (see Fig. 2), at a sufficiently small distance $2 a$, such that the electrons can tunnel between the dots. The tunnel junction between the dots, as well as the number of electrons on each dot, is controlled by the depleting gates (see Fig. 1). We consider the case of similar dots, and each dot contains an odd number of electrons with a spin 1/2 ground state. Furthermore, we simplify our consideration by retaining only one electron per dot and assuming that the rest of the electrons form a closed shell and merely contribute to the confining potential of the gates. We model the two coupled dots with the Hamiltonian 39] $H=\sum_{i=1,2} h_{i}+C+H_{\mathrm{Z}}=H_{\text {orb }}+H_{\mathrm{Z}}$, where the single-electron dynamics in the 2DEG ( $x y$-plane) is described through

$$
h_{i}=\frac{1}{2 m}\left(\mathbf{p}_{i}-\frac{e}{c} \mathbf{A}\left(\mathbf{r}_{i}\right)\right)^{2}+W\left(\mathbf{r}_{i}\right),
$$

with $m$ being the effective mass and $W\left(\mathbf{r}_{i}\right)$ the confinement potential as given below. A magnetic field $\mathbf{B}=(0,0, B)$ is applied along the $z$-axis, which couples to the electron spin through the Zeeman interaction $H_{\mathrm{Z}}$ and to the charge through the vector potential $\mathbf{A}(\mathbf{r})=\frac{B}{2}(-y, x, 0)$. In almost depleted regions, like fewelectron quantum dots, the screening length $\lambda$ can be expected to be much larger than the screening length in bulk 2DEG regions (where it is $40 \mathrm{~nm}$ for GaAs). Thus, for small quantum dots, say $\lambda \gg 2 a \approx 40 \mathrm{~nm}$, we need to consider the bare Coulomb interaction $C=e^{2} / \kappa\left|\mathbf{r}_{1}-\mathbf{r}_{2}\right|$, where $\kappa$ is the static dielectric constant. The confinement and tunnel-coupling in Eq. (4) for laterally aligned dots is modeled by the quartic potential

$$
W(x, y)=\frac{m \omega_{0}^{2}}{2}\left[\frac{1}{4 a^{2}}\left(x^{2}-a^{2}\right)^{2}+y^{2}\right]
$$

with the inter-dot distance $2 a$ and $a_{\mathrm{B}}=\sqrt{\hbar / m \omega_{0}}$ the effective Bohr radius of the dot. Separated dots $\left(a \gg a_{\mathrm{B}}\right)$ are thus modeled as two harmonic wells with frequency $\omega_{0}$. This is motivated by the experimental evidence that the low-energy spectrum of single dots is well described by a parabolic confinement potential 28].

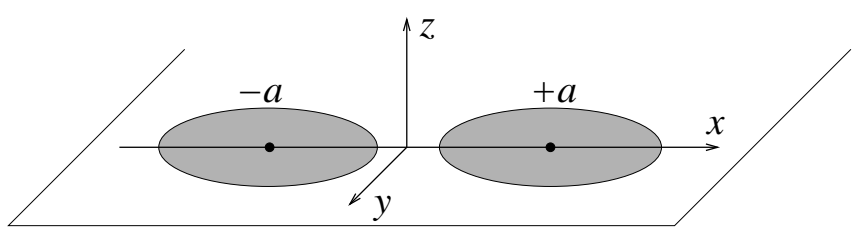

FIG. 2. Double dot geometry

Now we first classify the two-particle states according to the available symmetries, and then we calculate the phenomenological parameters within our toy model. The wave function of the two electrons in the double dot (DD) can be chosen to be a product of an orbital and a spin part. Then, in a singlet (triplet) state the orbital part is symmetric (antisymmetric) with respect to the interchange of the electrons, while the spin part is antisymmetric (symmetric). With respect to the mirror reflection in the $y z$-plane [45] (see Fig. 2) the singleparticle states fall into two symmetries, which we label by the quantum number $n= \pm$. The energy difference between the state with $n=-$ and that with $n=+$ is given by $2 t_{0}>0\left(t_{0}\right.$ being the interdot hopping amplitude). Within the low energy sector, the lowest singlet state and the triplet states are then given by 46.

$$
\begin{aligned}
& |00\rangle=\frac{1}{\sqrt{1+\phi^{2}}}\left(d_{+\uparrow}^{\dagger} d_{+\downarrow}^{\dagger}-\phi d_{-\uparrow}^{\dagger} d_{-\downarrow}^{\dagger}\right)|0\rangle, \\
& |11\rangle=d_{-\uparrow}^{\dagger} d_{+\uparrow}^{\dagger}|0\rangle, \quad|1-1\rangle=d_{-\downarrow}^{\dagger} d_{+\downarrow}^{\dagger}|0\rangle, \\
& |10\rangle=\frac{1}{\sqrt{2}}\left(d_{-\uparrow}^{\dagger} d_{+\downarrow}^{\dagger}+d_{-\downarrow}^{\dagger} d_{+\uparrow}^{\dagger}\right)|0\rangle
\end{aligned}
$$

where the notation $\left|S S_{z}\right\rangle$ stands for the angular momentum representation of the total spin of the two electrons. Here, the second quantized operator $d_{n \sigma}^{\dagger}$ creates an electron in the orbital state $n$ with spin $\sigma$. The vacuum state $|0\rangle$ includes the disregarded electrons. The interaction parameter $\phi$ depends on the interplay between the tunneling and Coulomb interaction. We have calculated $\phi$ 47] within the Hund-Mulliken method, 


$$
\phi=\sqrt{1+\left(\frac{4 t_{H}}{U_{H}}\right)^{2}}-\frac{4 t_{H}}{U_{H}},
$$

where $t_{H}$ and $U_{H}$ are the extended inter-dot tunneling amplitude and on-site Coulomb repulsion, respectively [39. We note that $t_{H}=t_{0}+t_{C}$, with the contribution $t_{C}$ coming from the Coulomb interaction [39] and vanishing with vanishing $t_{0}$. For detached dots we have $\phi=1$, and $\phi<1$ occurs due to double occupancies in the dots, and $\phi \rightarrow 0$ for vanishing Coulomb interaction. By varying $a$, we plot $\phi$ versus $t_{0}$ on Fig. 3. Next, we note that the singlet state in (6) represents an entangled state of two electrons, with the entanglement being

$$
\eta=\frac{2 \phi}{1+\phi^{2}} .
$$

Here, we used the measure of entanglement introduced in Ref. [36].

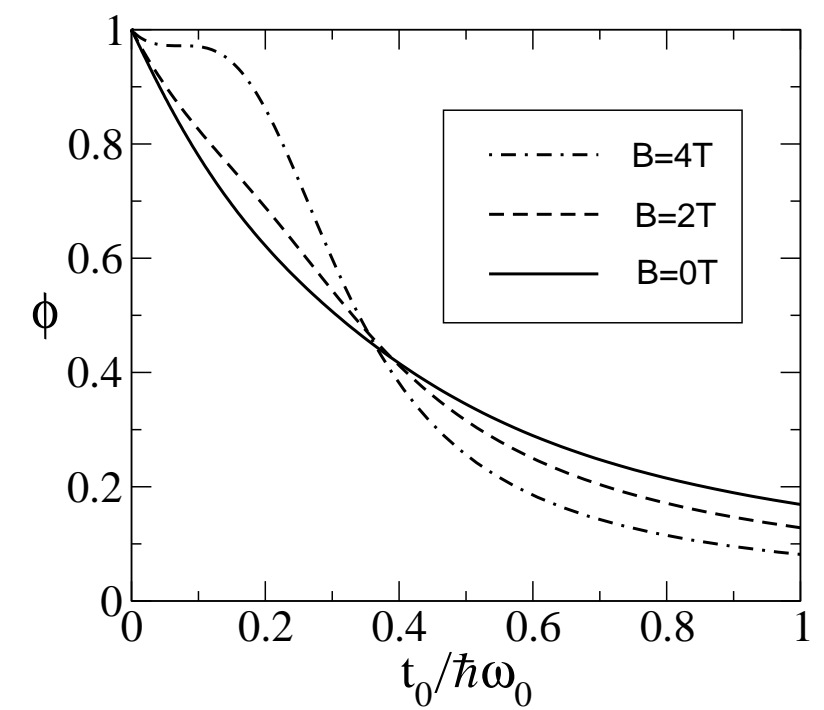

FIG. 3. Parameter $\phi$ versus the interdot hopping amplitude $t_{0}$ at different magnetic fields (Hund-Mulliken calculation). We used GaAs quantum dots with confinement energy $\hbar \omega_{0}=3 \mathrm{meV}$ and dielectric constant $\kappa=13.1$.

Next, assuming adiabatic switching of the coupling constants, we arrive at the effective Hamiltonian (2) by means of the following mapping [48,47

$$
\begin{aligned}
& \sum_{\sigma \sigma^{\prime}} d_{n \sigma}^{\dagger} \boldsymbol{\sigma}_{\sigma \sigma^{\prime}} d_{n^{\prime} \sigma^{\prime}}=\mathbf{S}_{+} \delta_{n n^{\prime}}+\left(\frac{\phi_{+}}{2} \mathbf{S}_{-}+i n \phi_{-} \mathbf{T}\right) \delta_{-n n^{\prime}} \\
& \sum_{\sigma} d_{n \sigma}^{\dagger} d_{n^{\prime} \sigma}=\delta_{n n^{\prime}}\left[1-\frac{n}{2} \phi_{+} \phi_{-}\left(\mathbf{S}_{1} \cdot \mathbf{S}_{2}-\frac{1}{4}\right)\right]
\end{aligned}
$$

where $\mathbf{S}_{ \pm}=\mathbf{S}_{1} \pm \mathbf{S}_{2}, \mathbf{T}=\mathbf{S}_{1} \times \mathbf{S}_{2}, \phi_{ \pm}=\sqrt{2}(1 \pm$ $\phi) / \sqrt{\left(1+\phi^{2}\right)}$, and $\boldsymbol{\sigma}$ are the Pauli matrices. Mapping (9) projects out the higher energy sector of the DD and keeps only the states (6). We note that the spin $1 / 2$ operators $\mathbf{S}_{1,2}$ are nothing but the intermixed electron spins, and hence represent the spin degrees of freedom of the DD. When detaching the two dots adiabatically, one always obtains one electron in each dot [36] and the spins $\mathbf{S}_{1,2}$ then stand for the true electron spins. Also note that during an adiabatic coupling of the two qubits (dots) each of the spins $\mathbf{S}_{1,2}$ carries the initial information of its qubit, which gets modified only through the Heisenberg exchange interaction $J$, see Eq. (2). The adiabaticity criterion discussed in Sec. II C applies hence here with $\delta \varepsilon=\min \left(\hbar \omega_{0}, U_{H}\right)$.

The Heisenberg exchange interaction $J$, which is defined as the energy difference between the triplet and singlet states (6), is the only parameter of interest for the two-qubit dynamics (provided the adiabaticity criterion is fulfilled). Although for a real structure it is best to have methods to measure $J$ for different values of the gate voltages, i.e. to characterize the structure experimentally (see Sec. VI), we still find it instructive to analyse various contributions to $J$ within our realistic model of the DD. In particular, we show that breaking the time-reversal symmetry by means of applying a magnetic field leads to a singlet-triplet transition in the DD. We calculate $J$ using different methods and compare the results. A generic expression for $J$ is straightforwardly obtained from the states (6),

$$
J=V+\frac{1-\phi^{2}}{1+\phi^{2}} 2 t_{H}-\frac{(1-\phi)^{2}}{1+\phi^{2}} \frac{U_{H}}{2}
$$

with $V=\langle T|C| T\rangle-\left\langle 00^{+}|C| 00^{+}\right\rangle, \quad t_{H}=t-$ $\left\langle 00^{+}|C| 00^{-}\right\rangle / 2$, and $U_{H}=\left\langle 00^{-}|C| 00^{-}\right\rangle-\left\langle 00^{+}|C| 00^{+}\right\rangle$. Here, $|T\rangle$ stands for any of the triplet states in (6), and $\left|00^{ \pm}\right\rangle$denotes the singlet state of (6) taken at $\phi= \pm 1$. In the Hund-Mulliken approach, $\phi$ is given by (7) and expressions for $V, t_{H}$, and $U_{H}$ were obtained in Ref. [39]. The Heisenberg exchange interaction then reads

$$
J=V-\frac{U_{H}}{2}+\frac{1}{2} \sqrt{U_{H}^{2}+16 t_{H}^{2}},
$$

We note that the component $V$ is responsible for making $J$ ferromagnetic, i.e. $J<0$. In the standard Hubbard approach for short-range Coulomb interaction it is assumed that there is no overlap between the electron wave function on different dots, though there is a finite hopping amplitude $t$ (chain model). The exchange terms of the Coulomb interaction hence vanish, and this corresponds to setting $V \rightarrow 0, t_{H} \rightarrow t$, and $U_{H} \rightarrow U$, with $U$ being the on-site (short-range) Coulomb repulsion [39]. The parameter $\phi$ is given by a formula analogous to (7). The Heisenberg exchange interaction is then always antiferromagnetic, $J=\sqrt{(U / 2)^{2}+4 t^{2}}-U / 2>0$. Finally, in the Heitler-London approach, the double occupancy of each dot is neglected, which corresponds to setting $\phi=(1-S) /(1+S)$, where $S$ is the overlap integral between the electron wave functions on the two dots. Note that the Heitler-London method gives qualitatively wrong results for both the case of strong and 
weak Coulomb interaction, since the interaction parameter $\phi$ is not sensitive to the Coulomb interaction. However, for intermediate strengths of the Coulomb interaction $\left(U_{H} \sim \hbar \omega_{0}\right)$ it gives qualitatively correct results. The parameters $V, t_{H}$, and $U_{H}$ are identical for both Heitler-London and Hund-Mulliken methods. We find it appropriate to use the Heitler-London approximation for simplicity in a GaAs system. Formula (10) then reduces to [39],

$$
\begin{aligned}
J= & \frac{\hbar \omega_{0}}{\sinh \left(2 d^{2} \frac{2 b-1}{b}\right)}\left\{\frac{3}{4 b}\left(1+b d^{2}\right)\right. \\
& \left.+c \sqrt{b}\left[e^{-b d^{2}} I_{0}\left(b d^{2}\right)-e^{d^{2}(b-1) / b} I_{0}\left(d^{2} \frac{b-1}{b}\right)\right]\right\}
\end{aligned}
$$

where we have introduced the dimensionless distance $d=a / a_{\mathrm{B}}$ between the dots and the magnetic compression factor $b=B / B_{0}=\sqrt{1+\omega_{L}^{2} / \omega_{0}^{2}}$ with the Larmor frequency $\omega_{L}=e B / 2 m c$. The zeroth order Bessel function is denoted by $I_{0}$. In Eq. (12), the first term comes from the confinement potential, while the terms proportional to the parameter $c=\sqrt{\pi / 2}\left(e^{2} / \kappa a_{\mathrm{B}}\right) / \hbar \omega_{0}$ result from the Coulomb interaction $C$; the exchange term is recognized by its negative sign. We plot $J$ [Eq. (12)] in Fig. 田 as a function of $B$ and $d$. We note that $J(B=0)>0$, which is generally true for a two-particle system with timereversal invariance. We observe that over a wide range of the parameters $c$ and $a$, the sign of $J(B)$ changes from positive to negative at a finite value of $B$ (for the parameters chosen in Fig. $4(\mathrm{a})$ at $B \approx 1.3 \mathrm{~T}) . J$ is suppressed exponentially either by compression of the electron orbitals through large magnetic fields $(b \gg 1)$, or by large distances between the $\operatorname{dots}(d \gg 1)$, where in both cases the orbital overlap of the two dots is reduced. This exponential suppression, contained in the $1 / \sinh$ prefactor in Eq. (12), is partly compensated by the exponentially growing exchange term $\propto \exp \left(2 d^{2}(b-1 / b)\right)$. In total, $J$ decays exponentially as $\exp \left(-2 d^{2} b\right)$ for large $b$ or $d$. Since the sign reversal of $J$-signalling a singlet-triplet crossing - results from the long-range Coulomb interaction, it is not contained in the standard Hubbard model which takes only short-range interaction into account. Fig. 4 compares the results of different methods.

We remark again that the exponential suppression of $J$ is very desirable for minimizing gate errors. In the absence of tunneling between the dots we still might have direct Coulomb interaction left between the electrons. However, this has no effect on the spins (qubit) provided the spin-orbit coupling is sufficiently small, which is the case for s-wave electrons in GaAs structures with unbroken inversion symmetry (this would not be so for hole-doped systems since the hole has a much stronger spin-orbit coupling due to its p-wave character). For a detailed discussion of spin-orbit interaction and its effect on exchange and XOR gates we refer to Ref. [49].
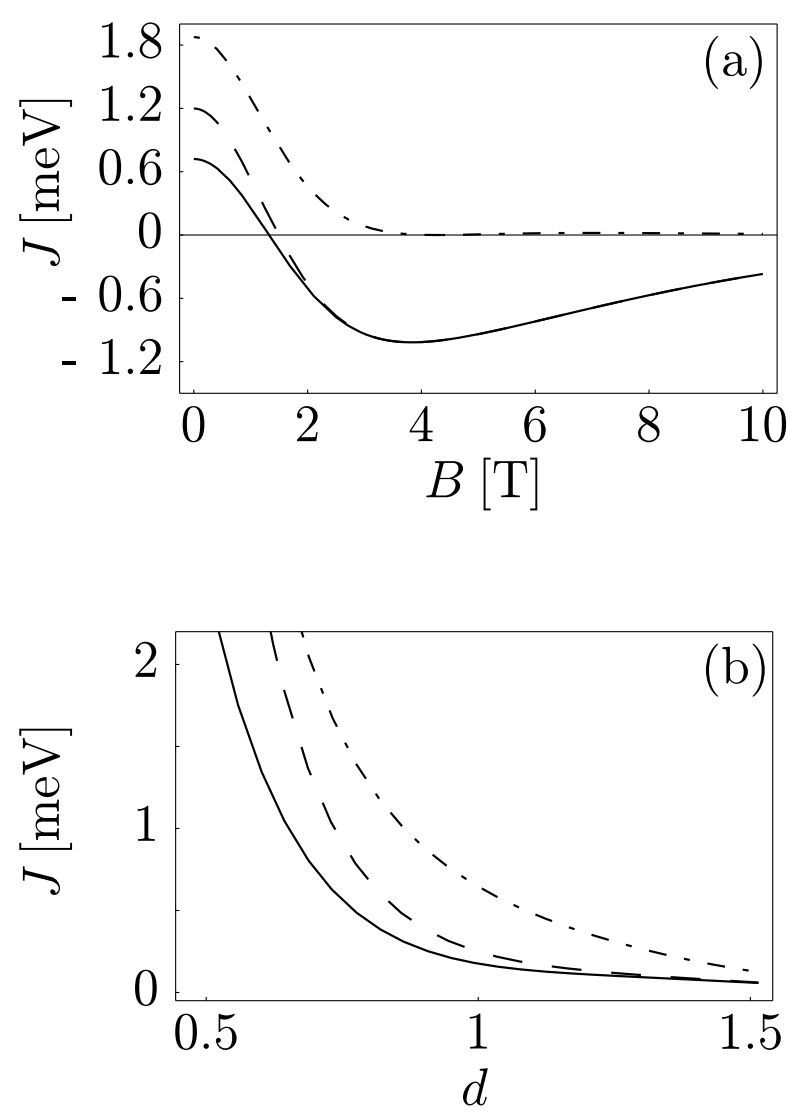

FIG. 4. Exchange coupling $J$ (full line) for GaAs quantum dots with confinement energy $\hbar \omega=3 \mathrm{meV}$ and $c=2.42$. For comparison we plot the usual short-range Hubbard result $J=4 t^{2} / U$ (dashed-dotted line) and the extended Hubbard result 39] $J=V+4 t_{H}^{2} / U_{H}$ (dashed line). In (a), $J$ is plotted as a function of the magnetic field $B$ at fixed inter-dot distance $d=a / a_{\mathrm{B}}=0.7$, while in (b) as a function of the inter-dot distance $d=a / a_{\mathrm{B}}$ at $B=0$.

\section{MEASURING A SINGLE SPIN (READ-OUT)}

\section{A. Spin Measurements through Spontaneous Magnetization}

One scheme for reading out the spin of an electron on a quantum dot is implemented by tunneling of this electron into a supercooled paramagnetic dot [8]. There the spin induces a magnetization nucleation from the paramagnetic metastable phase into a ferromagnetic domain, whose magnetization direction $(\theta, \varphi)$ is along the measured spin direction and which can be measured by conventional means. Since this direction is continuous rather than only one of two values, we describe this generalized measurement in the formalism of positiveoperator-valued (POV) measurements [50] as projection into the overcomplete set of spin- $1 / 2$ coherent states $|\theta, \varphi\rangle=\cos (\theta / 2)|\uparrow\rangle+e^{i \varphi} \sin (\theta / 2)|\downarrow\rangle$. Thus if we interpret a magnetization direction in the upper hemi- 
sphere as $|\uparrow\rangle$, we have a $75 \%$-reliable measurement, since $(1 / 2 \pi) \int_{\theta \geq \pi / 2} d \Omega|\langle\uparrow \mid \theta, \varphi\rangle|^{2}=3 / 4$, using the normalization constant $2 \pi$ for the coherent spin states.

\section{B. Quantum Dot as Spin Filter and Read-Out/Memory Device}

We discuss now a setup - quantum dot attached to inand outgoing current leads $l=1,2$ - which can be operated as a spin filter, or as a read-out device, or as a spinmemory where a single spin stores the information [9].

A new feature of this proposal is that the spindegeneracy is lifted with different Zeeman splittings in the dot and in the leads, e.g. by using materials with different effective g-factors for leads and dot [9]. This results in Coulomb blockade peaks and spin-polarized currents which are uniquely associated with the spin state on the dot.

The setup is described by a standard tunneling Hamiltonian $H_{0}+H_{T}$ [51], where $H_{0}=H_{L}+H_{D}$ describes the leads and the dot. $H_{D}$ includes the charging and interaction energies of the electrons in the dot as well as their Zeeman energy $\pm g \mu_{B} B / 2$ in an external magnetic field B. The tunneling between leads and the dot is described by $H_{T}=\sum_{l, k, p, s} t_{l p} c_{l k s}^{\dagger} d_{p s}+$ h.c., where $c_{l k s}$ annihilates electrons with spin $s$ and momentum $k$ in lead $l$, and $d_{p s}$ annihilates electrons in the dot. We consider the Coulomb blockade regime [1] where the charge on the dot is quantized. Then we apply a standard master-equation approach 52,9] with a reduced density matrix of the dot and calculate the transition rates in a "golden-rule" approach up to 2 nd order in $H_{T}$. The first-order contribution to the current is the sequential tunneling current $I_{s}$ [1], where the number of electrons on the dot fluctuates and thus the processes of an electron tunneling from the lead onto the dot and vice versa are allowed by energy conservation. The second-order contribution is the cotunneling current $I_{c}$ [53], involving a virtual intermediate state with a different number of electrons on the dot.

We now consider a system, where the Zeeman splitting in the leads is negligible (i.e. much smaller than the Fermi energy), while on the dot it is given as $\Delta_{z}=\mu_{B}|g B|$. We assume a small bias $\Delta \mu=\mu_{1}-\mu_{2}>0$ between the leads at chemical potential $\mu_{1,2}$ and low temperatures so that $\Delta \mu, k T<\delta$, where $\delta$ is the characteristic energy-level distance on the dot. First we consider a quantum dot in the ground state, filled with an odd number of electrons with total spin $1 / 2$, which we assume to be $|\uparrow\rangle$ and to have energy $E_{\uparrow}=0$. If an electron tunnels from the lead onto the dot, a spin singlet is formed with energy $E_{S}$, while the spin triplets are (usually) excited states with energies $E_{T_{ \pm}}$and $E_{T_{0}}$. At the sequential tunneling resonance, $\mu_{1}>E_{S}>\mu_{2}$, where the number of electrons on the dot fluctuates between $N$ and $N+1$, and in the regime $E_{T_{+}}-E_{S}, \Delta_{z}>\Delta \mu, k T$, energy conservation only allows ground state transitions. Thus, spin-up electrons are not allowed to tunnel from lead 1 via the dot into lead 2, since this would involve virtual states $\left|T_{+}\right\rangle$ and $|\downarrow\rangle$, and so we have $I_{s}(\uparrow)=0$ for sequential tunneling. However, spin down electrons may pass through the dot in the process $\left.\downarrow \uparrow{ }_{i} \rightarrow \uparrow \downarrow\right)_{f}$, followed by $\uparrow \downarrow_{i} \rightarrow$ $\uparrow \downarrow_{f}$. Here the state of the quantum dot is drawn inside the circle, while the states in the leads are drawn to the left and right, resp., of the circle. This leads to a spinpolarized sequential tunneling current $I_{s}=I_{s}(\downarrow)$, which we have calculated as [9]

$$
\begin{array}{ll}
I_{s}(\downarrow) / I_{0}=\theta\left(\mu_{1}-E_{S}\right)-\theta\left(\mu_{2}-E_{S}\right), & k_{B} T<\Delta \mu, \\
I_{s}(\downarrow) / I_{0}=\frac{\Delta \mu}{4 k_{B} T} \cosh ^{-2}\left[\frac{E_{S}-\mu}{2 k_{B} T}\right], & k_{B} T>\Delta \mu,
\end{array}
$$

where $\mu=\left(\mu_{1}+\mu_{2}\right) / 2$ and $I_{0}=e \gamma_{1} \gamma_{2} /\left(\gamma_{1}+\gamma_{2}\right)$. Here $\gamma_{l}=2 \pi \nu\left|A_{l n n^{\prime}}\right|^{2}$ is the tunneling rate between lead $l$ and the dot, and we have introduced the matrix elements $A_{l n^{\prime} n}=\sum_{p s} t_{l p}\left\langle n^{\prime}\left|d_{p s}\right| n\right\rangle$. Similarly, for $N$ even we find $I_{s}(\downarrow)=0$, while for $I_{s}(\uparrow)$ a similar result holds [9] as in Eqs. (13), (14).

Even though $I_{s}$ is completely spin-polarized, a leakage of current with opposite polarization arises through cotunneling processes [9]; still the leakage is small, and the efficiency for $\Delta_{z}<\left|E_{T_{+}}-E_{S}\right|$ for spin filtering in the sequential regime becomes [9] (for $\gamma_{1} \sim \gamma_{2}$ )

$$
I_{s}(\downarrow) / I_{c}(\uparrow) \sim \frac{\Delta_{z}^{2}}{\left(\gamma_{1}+\gamma_{2}\right) \max \left\{k_{B} T, \Delta \mu\right\}},
$$

and equivalently for $I_{s}(\uparrow) / I_{c}(\downarrow)$ at the even-to-odd transition. In the sequential regime we have $\gamma_{i}<k_{B} T, \Delta \mu$, thus, for $k_{B} T, \Delta \mu<\Delta_{z}$, we see that the spin-filtering is very efficient.

We discuss now the opposite case where the leads are fully spin polarized with a much smaller Zeeman splitting on the dot [9]. Such a situation can be realized with magnetic semiconductors (with effective g-factors reaching 100 [4) where spin-injection into GaAs has recently been demonstrated for the first time [4].5]. Another possibility would be to work in the quantum Hall regime where spin-polarized edge states are coupled to a quantum dot [54]. In this setup the device can be used as read-out for the spin state on the dot. Assume now that the spin polarization in both leads is up, and the ground state of the dot contains an odd number of electrons with total spin $1 / 2$. Now the leads can provide and absorb only spin-up electrons. Thus, a sequential tunneling current will only be possible if the dot state is $|\downarrow\rangle$ (to form a singlet with the incoming electron, whereas the triplet is excluded by energy conservation). Hence, the current is much larger for the spin on the dot being in $|\downarrow\rangle$ than it is for $|\uparrow\rangle$. Again, there is a small cotunneling leakage current for the dot-state $|\uparrow\rangle$, with a ratio of the two currents given by Eq. (15) with $\Delta_{z}$ replaced by $E_{T+}-E_{S}$. Thus, we can probe (read out) the spin-state on the quantum dot by measuring the current which passes through the dot. 
Given that the sequential tunneling current is typically on the order of $0.1-1 \mathrm{nA}$ [1], we can estimate the readout frequency $I / 2 \pi e$ to be on the order of $0.1-1 \mathrm{GHz}$. Combining this with the initialization and read-in techniques, i.e. ESR pulses to switch the spin state, we have a spin memory at the ultimate single-spin limit, whose relaxation time is just the spin relaxation time $T_{1}$. This relaxation time can be expected to be on the order of 100's of nanoseconds [3], and can be directly measured via the currents when they switch from high to low due to a spin flip on the dot 99. Furthermore, the spin decoherence time $T_{2}$ can also be measured via the current, if an ESR field is applied to the dot in either sequential tunneling or cotunneling regime with normal (unpolarized) leads, as shown in Ref. 27.

\section{ACCESSING THE SINGLET-TRIPLET SPLITTING IN DOUBLE DOTS}

Transport measurements can be used to characterize a quantum dot system experimentally. A main parameter of interest for quantum computing is the exchange interaction $J$ between the spins of two neighboring dots. We have considered a setup [47, consisting of two lateral quantum dots connected in series between two metallic leads, see Fig. 5. A magnetic field $B$, applied perpendicular to the plane of the dots, is used to tune the exchange interaction $J$. A common gate (not shown), with the gate voltage $V_{g}$, can be used to change the electron occupation number of the double dot (DD). The conductance of the DD versus $V_{g}$ shows peaks of sequential tunneling separated by Coulomb blockade valleys [here we consider temperatures smaller than the Coulomb correlation energy]. We focus on the valley with two electrons in the DD. Our consideration also holds for a larger ocupation number $M=2 N$, with $N$ being odd, provided $N-1$ electrons on each dot form a closed shell and can be disregarded. The two (outer shell) electrons are confined by the DD potential, however their spin degrees of freedom can be correlated on a much smaller energy scale $J$. Our aim is to provide ways of accessing the exchange interaction $J$ between the spins of the two electrons in the DD.

Readily the differential cotunneling conductance through the DD shows distinct features (steps at the bias $\Delta \mu= \pm J$ ), which allow one to measure $J$ experimentally. However, attaching leads to the DD shifts the energy levels, and hence, modifies $J$. Moreover, measuring a small value of $J$ requires low temperatures at which the Kondo correlations in the leads may be important. We show that such Kondo correlations also introduce a correction to $J$, which is temperature dependent. We find that the peculiar features in transport properties are better pronounced in the Kondo regime. For example, the linear conductance of the DD as function of temperature shows a maximum at temperature $T \simeq J>0$, which is pronounced only in the Kondo regime. This maximum can be used as an alternative way of measuring $J$, having the advantage that, in the linear regime, the DD is not affected by the applied bias.

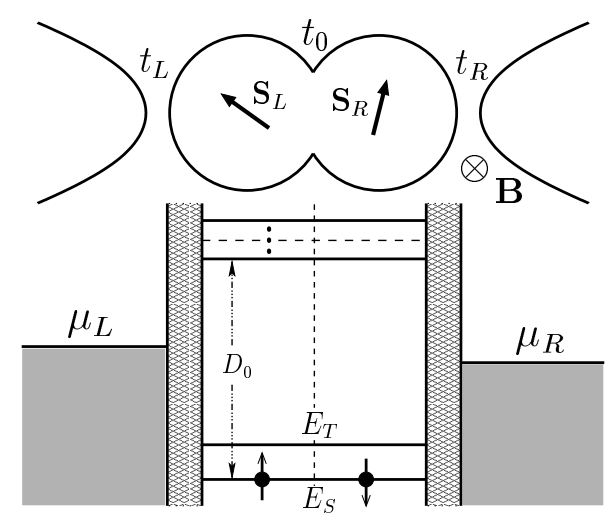

FIG. 5. Double-dot system containing two electrons and being coupled in series to two metallic leads at chemical potentials $\mu_{R}$ and $\mu_{L}$ with bias $\Delta \mu=\mu_{L}-\mu_{R}$. The electron spins $\mathbf{S}_{L}, \mathbf{S}_{R}$ interact via the exchange interaction $J=E_{T}-E_{S}$, where $E_{T, S}$ is the triplet/singlet energy.

\section{A. Cotunneling through two tunnel-coupled quantum dots}

In the Coulomb blockade regime, the fluctuations of the number of electrons on the DD are strongly suppressed by the Coulomb blockade gap. The conductance through the DD is dominated by processes with a virtual occupation of the DD by a lead electron (hole), i.e. cotunneling processes. We consider a realistic DD with long range Coulomb interaction between the two electrons on the DD, as discussed in Sec. IVA. Using a Hund-Mulliken approach, we write down the one-electron states as $\psi_{ \pm, \sigma}=\chi_{\sigma}\left(\varphi_{-a} \pm \varphi_{+a}\right) / \sqrt{2(1 \pm S)}$, where $\chi_{\sigma}$ is the spinor, $\varphi_{ \pm a}$ are the lowest orbitals of single dots situated at $x= \pm a$, and $S=\left\langle\varphi_{ \pm a} \mid \varphi_{\mp a}\right\rangle$ is the overlap integral. The lowest in energy singlet and triplet are given then by Eq. (6), and the interaction parameter $\phi$ is calculated according to (7). The attached leads are described by $H_{l}=\sum_{\alpha k \sigma} \varepsilon_{k} c_{\alpha k \sigma}^{\dagger} c_{\alpha k \sigma}$, where $c_{\alpha k \sigma}^{\dagger}$ creates an electron with momentum $k$ and spin $\sigma$ in lead $\alpha=L, R$. The tunneling between the DD and the leads is described by $H_{T}=\sum_{n \alpha k \sigma}\left(t_{\alpha n} c_{\alpha k \sigma}^{\dagger} d_{n \sigma}+\right.$ h.c.). Here, $d_{n \sigma}$ annihilates an electron in the state $\psi_{n \sigma}$. The tunneling amplitudes are given by $t_{L, \pm}=t_{L} / \sqrt{2(1 \pm S)}$ and $t_{R, \pm}= \pm t_{R} / \sqrt{2(1 \pm S)}$, with $t_{\alpha}$ being the apmlitude to tunnel from lead $\alpha$ onto the the adjusting dot at $t_{0}=0$. We map our problem onto a two-level system, with level 1 corresponding to the singlet state and level 2 to the three triplet states. The occupation probabilities of these two levels, $\rho_{1}$ and $\rho_{2}$, are given by

$$
\rho_{1}=\frac{1}{1+3 \exp \left(-J / T_{\text {eff }}\right)}=1-\rho_{2} .
$$


The effective temperature $T_{\text {eff }}$ depends on the applied bias $\Delta \mu$, and thus, describes the heating effects on the DD. Solving a master equation for $\rho_{1}$ and $\rho_{2}$ in the cotunneling regime, we find

$$
\frac{1}{T_{\mathrm{eff}}}=\frac{1}{T}-\frac{1}{J} \ln \frac{1+\lambda(J / T, \Delta \mu / T) \phi_{-}^{2}}{1+\lambda(-J / T, \Delta \mu / T) \phi_{-}^{2}},
$$

where $\lambda(u, v)=\frac{1}{4} \frac{\sinh (v)}{1-\tanh (u / 2)} \frac{\tanh (v / 2)-(v / u) \tanh (u / 2)}{\cosh (u)-\cosh (v)}$, and the parameter $\phi_{-}$was introduced in Sec. IVA. We note that the heating effect depends on the interaction parameter $\phi$, and for $\phi \rightarrow 1$ it vanishes. Also, the heating effect is pronounced only for biases $|\Delta \mu| \geq|J|$, and it vanishes at high temperatures. For the vicinity of $\Delta \mu= \pm J$, we define a characteristic temperature of a strong heating regime, given by $T_{\mathrm{h}}=|J| / w\left(8 / \phi_{-}^{2}\right)$, where the function $w(x)$ is defined for $x \geq e$ by $w(x)=\ln (x \ln (x \ldots))$. Bellow this temperature $T_{\mathrm{h}}$, the exponential dependence of $\exp \left(-J / T_{\text {eff }}\right)$ on $T$ is replaced by a power law dependence; the temperature $T$ competes with $|\Delta \mu|-|J|$, and as the latter becomes larger, the occupation probabilities cease to depend on $T$. For the strong heating regime $T<T_{\mathrm{h}}$, we find

$$
T_{\mathrm{eff}} \simeq \frac{|J|}{\ln \left(1+\frac{8}{\phi_{-}^{2}} \frac{|J|}{\max (T,|\Delta \mu|-|J|)}\right)}, \quad|\Delta \mu| \geq|J| .
$$

The current through the DD consists of an elastic and inelastic component, $I=I_{\mathrm{el}}+I_{\mathrm{inel}}$. In the middle of the Coulomb blockade valley the current components are given by

$$
\begin{aligned}
I_{\mathrm{el}}= & \frac{e}{h} \frac{2 \gamma^{2}}{\left(1-S^{2}\right)^{2}}\left[\phi_{-}^{2} \phi_{+}^{2} \rho_{1}+8 S^{2} \rho_{2}\right] \Delta \mu \\
I_{\text {inel }}= & \frac{e}{h} \frac{2 \gamma^{2} \phi_{-}^{2}}{1-S^{2}}\left\{[\Theta(-J+\Delta \mu)-\Theta(-J-\Delta \mu)] 3 \rho_{1}\right. \\
& \left.+[\Theta(J+\Delta \mu)-\Theta(J-\Delta \mu)] \rho_{2}\right\}
\end{aligned}
$$

where $\Theta(J)=J /(1-\exp (-J / T))$, and $\gamma=\pi \nu t_{L} t_{R} / E_{C}$, with $E_{C}$ being the Coulomb blockade half-gap, and $\nu$ the density of states in the leads. In the absence of heating, when $T_{\text {eff }}=T$, the elastic component $I_{\mathrm{el}}$ is linear in the applied bias $\Delta \mu$, and the inelastic one $I_{\text {inel }}$ exhibits a threshold-like switching-on at $|\Delta \mu|=|J|$, for $T<|J|[47]$. This results in steps in differential conductance versus $\Delta \mu$ at $|\Delta \mu|=|J|$, which can be used to measure the singlet-triplet splitting experimentally [55]. For $T \ll|J|$, the step height was found to be 3 times larger on the singlet side than on the triplet side 47. In the strong heating regime, the differential conductance $G=e d I / d \Delta \mu$ provides information also about the DD parameter $\phi$. We plot $G(\Delta \mu)$ in Fig. 6, for (a) a singlet and (b) a triplet ground state. The dashed line shows the result of a calculation where the heating effects are neglected. We find that, in the strong heating regime, $d I_{\text {inel }} / d \Delta \mu$ has a negative (positive) slope on the plateau $|\Delta \mu|>|J|$ for the ground state being a singlet (triplet).
The slope of the elastic component is not generic, but depends on the interplay between the parameters $S$ and $\phi$. However, we still find that the slope of the total conductance is not changed qualitatively by the elastic component over a large range of parameters calculated in the Hund-Mulliken method, Sec. IV A.
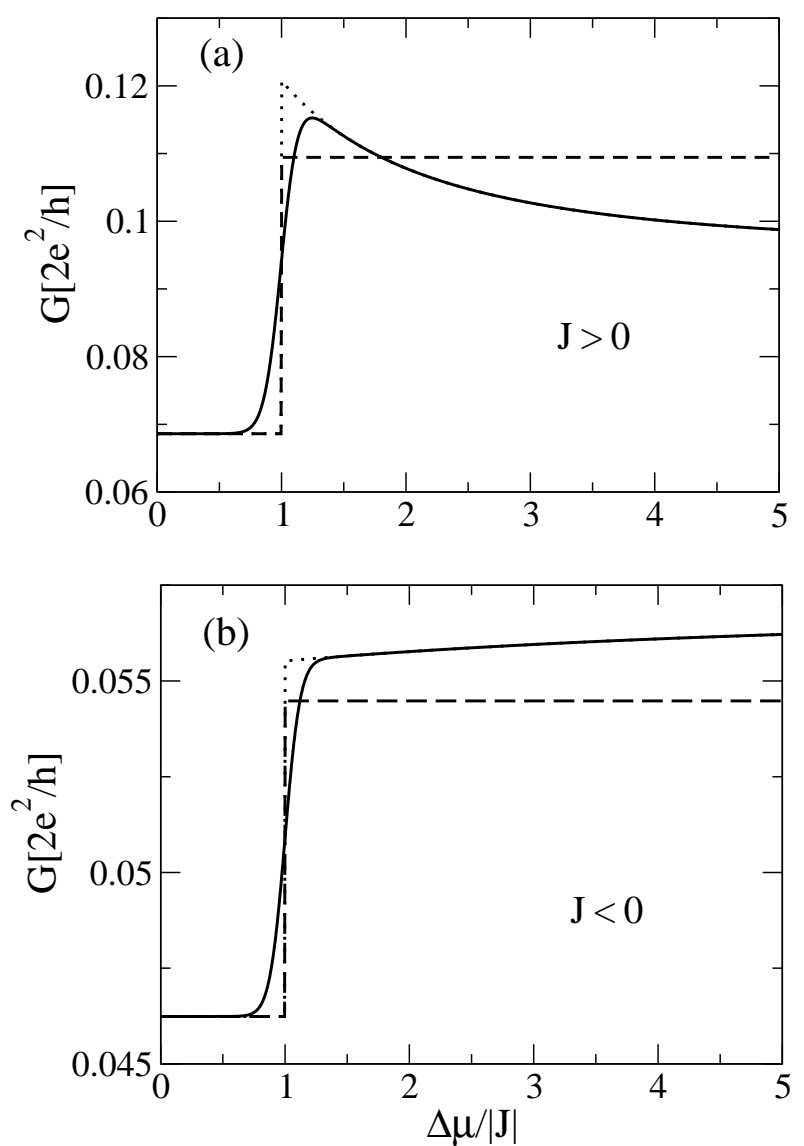

FIG. 6. Differential conductance $G=d I / d \Delta \mu$ versus bias calculated for dot separation $a / a_{B}=0.7$ and coupling to the leads given by $\gamma=0.1$ at a magnetic field: (a) $B=0$ and (b) $B=1.5 \mathrm{~T}$. The solid line and the dotted line are calculated in the strong heating regime at $T=0.2 T_{\mathrm{h}}$ and $T=0$, respectively. The dashed line corresponds to neglecting the heating effects, i.e. $T_{\text {eff }} \rightarrow T$, and was calculated for $T=0$. Thus we see that $G$ is monotonically increasing for $J<0$, while it has a maximum for $J>0$.

Next, we consider the case of weakly coupled $\operatorname{dots}\left(\phi_{-}^{2} \ll\right.$ 1 ) and show how the parameter $\phi$ can be extracted from $G(\Delta \mu)$ in the strong heating regime. For the singlet ground state, and the bias satisfying $T \ll|\Delta \mu|-|J| \ll$ $|J| / \phi_{-}^{2}$, we find

$$
\phi_{-}^{2}=\frac{A}{1-A \frac{|\Delta \mu|-|J|}{2|J|}}, \quad A \equiv-\frac{|J|}{\Delta G} \frac{d G}{d \Delta \mu},
$$

where $\Delta G=G(\Delta \mu)-G(\infty)$. For the triplet ground state one should replace $\phi_{-}^{2} \rightarrow \frac{1}{3} \phi_{-}^{2}$ in (21). In Fig. 7, we plot the rhs of the equation for $\phi_{-}^{2}$ in (21) at different temperatures. In the strong heating regime $\left(T \ll T_{\mathrm{h}}\right)$, the 
curve saturates at the value of $\phi_{-}^{2}$ with increasing bias. This behavior can be used to measure the interaction parameter $\phi$ experimentally. We note that one can reliably determine $G(\infty)$ experimentally, as the conductance at $\Delta \mu \gg|J| / \phi_{-}^{2}$, only if the sequential tunneling processes can be excluded. For the middle of the Coulomb blockade valley, we require $|J|+|\Delta \mu|<E_{C}$, to avoid sequential tunneling via the heated excited state [56]. Furthermore, we note that at low temperatures Kondo resonances can develop at $\Delta \mu= \pm J$, invalidating Eq. (21). However, one can avoid this problem by satisfying either $T \gg T_{K}$ or $|\Delta \mu|-|J| \gg T_{K}$, with $T_{K}$ being the energy scale of the Kondo resonance.

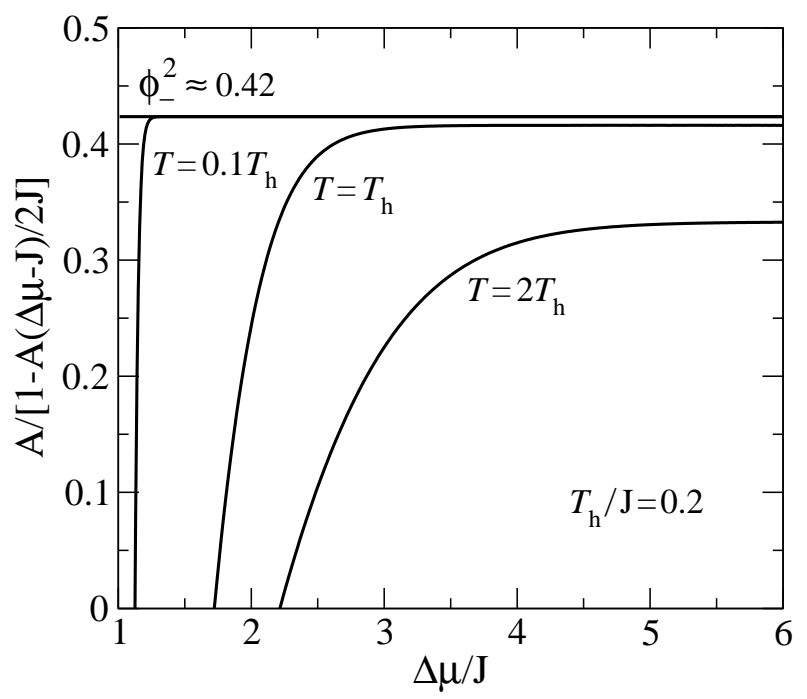

FIG. 7. A way to measure $\phi_{-}^{2}$ experimentally, see Eq. (21). For the calculation we used a dot separation of $a / a_{B}=1$ and $B=0$ (singlet ground state). Note that at $T>T_{\mathrm{h}}$ the lines saturate at a value which differs strongly from $\phi_{-}^{2}$.

\section{B. Kondo effect of two coupled dots at the singlet-triplet degeneracy point}

Attaching metallic leads to a DD can give rise to Kondo correlations at low temperatures. On the one hand, such correlations can enhance the conductance through a Coulomb-blockaded DD, making the transport measurements more accessible. On the other hand, Kondo correlations can modify the studied system and introduce a discrepancy between the measured values and the bare values of the system parameters. Knowledge of the Kondo effect in double dots would allow one to optimize the experimental setup, in order to obtain reliable data.

We adopted a "poor man's" scaling approach [57] to the DD system on Fig. 5 and obtained an effective Hamiltonian [47, describing the flow (with lowering $T$ ) into the Kondo regime of a DD at the singlet-triplet degeneracy point $J=0$. We found that the 4-fold degeneracy of the DD enhances the Kondo correlations on the Fermi surface, as compared to the case when the dots are detached from each other, or when the DD spins are locked into a spin 1 (triplet side). The Kondo temperature at $J=0$ is given by $47 T_{K}=D_{0} \exp \left(-\eta / \nu I_{0}\right)$, where $\eta \leq 0.5$ is a non-universal number dependent on the internal features of the DD, $D_{0} \simeq \hbar \omega_{0}$ is the cutoff energy (see Fig. 5), and $I_{0}=\left(t_{L}^{2}+t_{R}^{2}\right) / E_{C}$. We find that, at temperatures $T \lesssim T_{K}$, the DD undergoes a strong renormalization of its energy levels, resulting in a flow of the exchange interaction $J$ 47. Thus, we conclude that, at such temperatures and at $J \lesssim T_{K}$, the Kondo correlations strongly modify the coupling constant $J$, making any direct experimental measurement of the bare value of $J$ hardly possible. However, at larger values of $J>0$, one can make use of the Kondo correlations and still have a reliable measurement of $J$.

We calculated the current through the DD at a bias $\Delta \mu$, and found the renormalization of the linear conductance $G=\left.(d I / d \Delta \mu)\right|_{\Delta \mu=0}$ due to Kondo correlations. In Fig. 8 we plot the linear $G$ versus the inter-dot tunneling amplitude $t_{0}$ for different values of the magnetic field $B$. At $B=0$, the renormalized conductance (solid line) shows a sharp peak at a small value of $t_{0}$, owing to a competition between the Kondo effect of each dot with the adjusting lead and the antiferromagnetic exchange $J$. The peak position corresponds to $J(t) \simeq T_{K}$ [58]. At larger values of $t_{0}$, a second broader peak occurs, which is sensitive to applying a weak magnetic field, such that $J>0$. We find that the broad peak is present only if $J$ deviates from $4 t_{H}^{2} / U_{H}$ by the contribution $V$ (see Sec. [VA, which comes from the long range Coulomb interaction 39] (compare the solid lines with the dotdashed line in Fig. 8). Note that exactly this contribution to $J$ is responsible for the singlet-triplet transition in DDs. Thus, we have shown that the long range part of the Coulomb interaction can be probed experimentally in DDs, and screening effects can be studied.

On the left inset of Fig. 8, we plot the temperature dependence of the linear conductance calculated with taking into account the Kondo correlations (solid line) and neglecting them (dotted line), the latter corresponding to the cotunneling calculation of Sec. VIA. For the case with Kondo correlations, we find a pronounced maximum in the linear $G$ versus $T$ at $T \simeq J$, which can be used to estimate $J$ experimentally.

The $B$ dependence of the linear $G$ shows a peak at the singlet-triplet transition, which grows with lowering $T$ down to $T_{K}$ [47, see right inset of Fig. 8. Note that the enegry scale for the Kondo effect on the triplet side $(J<0)$ is monotonically decreasing with increasing $|J|$ [59,48], as follows from a two stage RG procedure valid on that side. Furthermore, the strong coupling limit (not shown in Fig. 8) occurs in two stages with lowering $T$ on the triplet side, resulting first in an increase and then, at a lower energy scale, in a decrease of the conductance 60. 


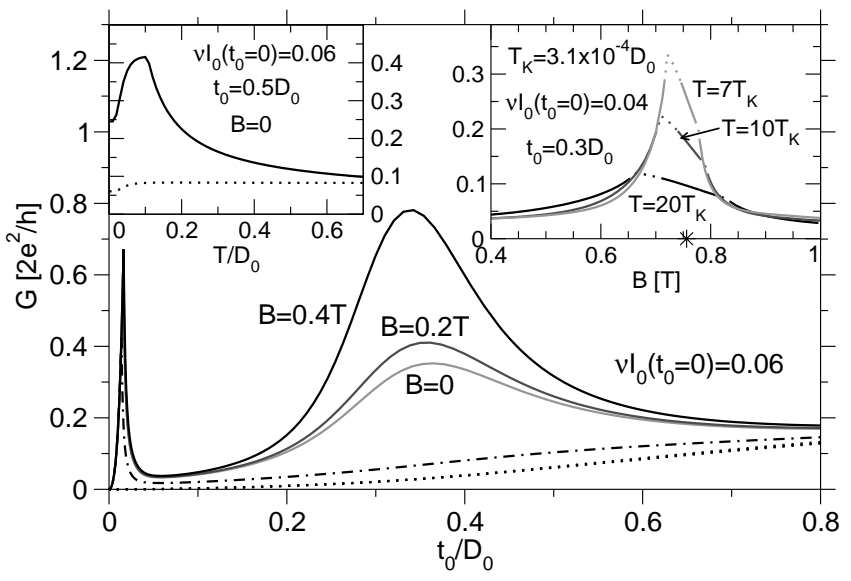

FIG. 8. Linear conductance $G$ at different values of $B$. Dotted lines: cotunneling contributions. Dot-dashed line: $G$ vs $t_{0}$ at $B=0.4 T$ neglecting the long range part of the Coulomb interaction. For definiteness we keep the DD in the middle of the Coulomb blockade valley by adjusting the gate voltage $V_{g}$ when varying $t_{0}$, and choose $t_{L}=t_{R}$. Left inset: $G$ vs $T$ showing a peak at $T \simeq J$; dotted line is the cotunneling contribution. Right inset: $G$ vs $B$ at the singlet-triplet transition; the kinks in the dotted-line regions come from a simplified treatment of the Kondo effect crossover regions and will be smoothened in an exact treatment; the star denotes the value of $B$ at which the singlet-triplet transition occurs at high temperatures $\left(T \gg T_{K}\right)$.

\section{CONCLUSIONS}

We have described a concept for a quantum computer based on electron spins in quantum-confined nanostructures, in particular quantum dots, and presented theoretical proposals for manipulation, coupling and detection of spins in such structures. We have discussed the requirements for coherence, switching times, read-out, gate operations and their actual realization. By putting it all together, we have illustrated how a scalable, allelectronically controlled quantum computer can be envisioned.

\section{ACKNOWLEDGMENTS}

We acknowledge support from the Swiss NSF, DARPA, and ARO.

[1] L. P. Kouwenhoven et al., Proceedings of the ASI on Mesoscopic Electron Transport, eds. L.L. Sohn, L.P. Kouwenhoven, and G. Schön (Kluwer, 1997).
[2] S.A. Wolf et al., Science 294, 1488 (2001).

[3] J.M. Kikkawa, I.P. Smorchkova, N. Samarth, and D.D. Awschalom, Science 277, 1284 (1997); J.M. Kikkawa and D.D. Awschalom, Phys. Rev. Lett. 80, 4313 (1998); D.D. Awschalom and J.M. Kikkawa, Phys. Today 52(6), 33 (1999).

[4] R. Fiederling et al., Nature 402, 787 (1999).

[5] Y. Ohno et al., Nature 402, 790 (1999).

[6] F.G. Monzon and M.L. Roukes, J. Magn. Magn. Mater. 198, 632 (1999).

[7] S. Lüscher et al., Phys. Rev. Lett. 86, 2118 (2001); condmat/0002226

[8] D. Loss and D.P. DiVincenzo, Phys. Rev. A 57, 120 (1998); cond-mat/9701055.

[9] P. Recher, E.V. Sukhorukov, and D. Loss, Phys. Rev. Lett. 85, 1962 (2000).

[10] M. A. Nielsen and I. L. Chuang, Quantum Computation and Quantum Information (Cambridge University Press, 2000).

[11] D.P. DiVincenzo and D. Loss, J. Magn. Magn. Mater. 200, 202 (1999); cond-mat/9901137.

[12] G. Burkard, D. Loss, and E.V. Sukhorukov, Phys. Rev. B, 61, R16303 (2000).

[13] P. Recher, E.V. Sukhorukov, and D. Loss, Phys. Rev. B 63, 165314 (2001).

[14] P.W. Shor, in Proc. 35th Symposium on the Foundations of Computer Science, (IEEE Computer Society Press), 124 (1994).

[15] L.K. Grover, Phys. Rev. Lett. 79, 325 (1997).

[16] C. H. Bennett and D. P. DiVincenzo, Nature 404, 247 (2000).

[17] J.I. Cirac and P. Zoller, Phys. Rev. Lett. 74, 4091 (1995); C. Monroe et al., ibid. 75, 4714 (1995).

[18] Q.A. Turchette et al., Phys. Rev. Lett. 75, 4710 (1995).

[19] D. Cory, A. Fahmy, and T. Havel, Proc. Nat. Acad. Sci. U.S.A. 94, 1634 (1997); N. A. Gershenfeld and I. L. Chuang, Science 275, 350 (1997).

[20] B. Kane, Nature 393, 133 (1998).

[21] A. Shnirman, G. Schön, and Z. Hermon, Phys. Rev. Lett. 79, 2371 (1997).

[22] D.V. Averin, Solid State Commun. 105, 659 (1998).

[23] L.B. Ioffe et al., Nature 398, 679 (1999).

[24] T.P. Orlando et al., Phys. Rev. B 60, 15398 (1999).

[25] V. Privman, I.D. Vagner, and G. Kventsel, Phys. Lett. A 239, 141 (1998).

[26] R. Vrijen et al., Phys. Rev. A 62, 012306 (2000).

[27] H.-A. Engel and D. Loss, Phys. Rev. Lett. 86, 4648 (2001); cond-mat/0109470.

[28] S. Tarucha et al., Phys. Rev. Lett. 77, 3613 (1996).

[29] F.R. Waugh et al., Phys. Rev. Lett. 75, 705 (1995); C. Livermore et al., Science 274, 1332 (1996).

[30] T. H. Oosterkamp et al., Phys. Rev. Lett. 80, 4951 (1998).

[31] R.H. Blick et al., Phys. Rev. Lett. 80, 4032 (1998); ibid. 81, 689 (1998). T.H. Oosterkamp et al., Nature 395, 873 (1998); I.J. Maasilta and V.J. Goldman, Phys. Rev. Lett. 84, 1776 (2000).

[32] J.A. Gupta, D.D. Awschalom, X. Peng, and A.P. Alivisatos, Phys. Rev. B 59, R10421 (1999). 
[33] D.P. DiVincenzo, G. Burkard, D. Loss, and E. Sukhorukov, in Quantum Mesoscopic Phenomena and Mesoscopic Devices in Microelectronics, eds. I.O. Kulik and R. Ellialtoglu (NATO ASI, Turkey, June 13-25, 1999); see cond-mat/99112445.

[34] A. Imamō̄lu et al., Phys. Rev. Lett. 83, 4204 (1999).

[35] M.-S. Choi, C. Bruder, and D. Loss, Phys. Rev. B 62, 13569 (2000).

[36] J. Schliemann, D. Loss, and A.H. MacDonald, Phys. Rev. B 63, 085311 (2001).

[37] P.W. Shor, Phys. Rev. A 52, R2493 (1995); A.M. Steane, Phys. Rev. Lett. 77, 793 (1996); D.P. DiVincenzo and P.W. Shor, ibid. 77, 3260 (1996); E. Knill and R. Laflamme, Phys. Rev. A 55, 900 (1997); D. Gottesman, ibid. 54, 1862 (1996); E. Dennis, Phys. Rev. A 63, 052314 (2001).

[38] J. Preskill, Proc. R. Soc. London Ser. A 454, 385 (1998); J. Preskill, in Introduction to Quantum Computation and Information, edited by H.-K. Lo, S. Popescu, and T. Spiller (World Scientific, Singapore, 1998), pp. 213269.

[39] G. Burkard, D. Loss, and D. P. DiVincenzo, Phys. Rev. B 59, 2070 (1999).

[40] R. Shankar, Principles of Quantum Mechanics, Ch. 14, Plenum Press, New York, 1994.

[41] E.L. Ivchenko, A.A. Kiselev, and M. Willander, Solid State Comm. 102, 375 (1997).

[42] G. Salis, et al., Nature 414, 619 (2001).

[43] D.P. DiVincenzo, Phys. Rev. A 51, 1015 (1995).

[44] A. Barenco et al., Phys. Rev. A 52, 3457 (1995).

[45] Note that, at $B \neq 0$, this implies also $B \rightarrow-B$.
[46] Note that $|00\rangle=|\Psi\rangle \otimes|\chi\rangle$, with the spin part $|\chi\rangle=(|\uparrow\rangle|\downarrow\rangle-|\downarrow\rangle|\uparrow\rangle) / \sqrt{2}$ and the orbital part $|\Psi\rangle=\left[\psi_{+}\left(\mathbf{r}_{1}\right) \psi_{+}\left(\mathbf{r}_{2}\right)-\phi \psi_{-}\left(\mathbf{r}_{1}\right) \psi_{-}\left(\mathbf{r}_{2}\right)\right] / \sqrt{1+\phi^{2}}$, where $\psi_{ \pm}(\mathbf{r})$ are the symetric/antisymmetric orbitals.

[47] V.N. Golovach and D. Loss, cond-mat/0109155.

[48] M. Pustilnik, L.I. Glazman, Phys. Rev. Lett. 85, 2993 (2000); Phys. Rev. B 64, 045328 (2001).

[49] G. Burkard, D. Loss, Phys. Rev. Lett. 88, 047903 (2002).

[50] A. Peres, Quantum Theory: Concepts and Methods (Kluwer, Dondrecht, 1993).

[51] G. D. Mahan, Many Particle Physics, 2nd Ed. (Plenum, New York, 1993).

[52] L.P. Kouwenhoven, G. Schön, and L. L. Sohn, Mesoscopic Electron Transport, NATO ASI Series E: Applied Sciences-Vol. 345 Kluwer Academic Publishers, 1997.

[53] D. V. Averin and Yu. V. Nazarov, in Single Charge Tunneling, eds. H. Grabert, M. H. Devoret, NATO ASI Series B: Physics Vol. 294, Plenum Press, New York, 1992.

[54] M. Ciorga et al., Phys. Rev. B 61, R16315 (2000).

[55] For single-dot experiment, see S. De Franceschi, et al., Phys. Rev. Lett. 86, 878 (2001).

[56] M.R. Wegewijs, Yu.V. Nazarov, cond-mat/0103579.

[57] P.W. Anderson, J. Phys. C 3, 2436 (1970); A.C. Hewson, The Kondo Problem to Heavy Fermions (Cambridge University Press, 1993).

[58] W. Izumida, O. Sakai, Phys. Rev. B 62, 10260 (2000), and references therein.

[59] M. Eto, Y. Nazarov, Phys. Rev. Lett. 85, 1306 (2000); Phys. Rev. B 64, 085322 (2001).

[60] M. Pustilnik, L.I. Glazman, Phys. Rev. Lett. 87, 216601 (2001). 